\title{
Une écriture en images : les armoiries parlantes
}

How to Write with Images? The Canting Arms

Michel Pastoureau

\section{CpenEdition}

Journals

Édition électronique

URL : http://journals.openedition.org/extremeorient/110

DOI : 10.4000/extremeorient. 110

ISSN : 2108-7105

Éditeur

Presses universitaires de Vincennes

Édition imprimée

Date de publication : 30 octobre 2008

Pagination : 187-198

ISBN : 978-2-84292-220-7

ISSN : 0754-5010

Référence électronique

Michel Pastoureau, « Une écriture en images : les armoiries parlantes », Extrême-Orient Extrême-

Occident [En ligne], 30 | 2008, mis en ligne le 01 octobre 2011, consulté le 06 mai 2019. URL : http:// journals.openedition.org/extremeorient/110 ; DOI : 10.4000/extremeorient.110 


\title{
Une écriture en images : les armoiries parlantes
}

\author{
Michel Pastoureau
}

Existe-t-il un langage des signes communs aux différentes civilisations? Probablement pas. Mais il existe, dans de nombreuses cultures, des signes visuels qui ont pour fonction de dire l'identité des individus et des groupes, et leur place dans la société. Ces signes s'expriment par des formules diverses et prennent place sur des supports de toutes natures: le corps, le vêtement et ses accessoires, les bijoux, les étoffes et les parures, la maison, le mobilier, les bâtiments et les monuments, les armes de guerre, les objets d'art et ceux de la vie quotidienne, les documents officiels et même les animaux domestiques ou le bétail. Certains, parmi ces signes, sont organisés en véritables systèmes et obéissent à des règles rigoureuses de composition, d'utilisation, de représentation et de transmission. D'autres sont d'un usage plus souple ou plus libre. Tous, ou presque, présentent la particularité d'être chargés d'une forte dimension symbolique et de toujours dire plus que ce qu'ils ont pour fonction de dire.

Les armoiries européennes comptent parmi ces signes. Elles peuvent se définir comme des emblèmes en couleurs, propres à un individu, à une famille ou à une communauté, et soumis dans leur composition et leur représentation à des règles particulières qui sont celles du blason. Ainsi définies, les armoiries apparaissent dans le courant du XII ${ }^{\mathrm{e}}$ siècle sur les champs de bataille et de tournoi, et s'organisent rapidement en un code social, à la fois ouvert et rigoureux: l'héraldique. C'est l'existence de quelques règles de composition - peu nombreuses, mais contraignantes - qui différencie le système héraldique européen de tous les autres systèmes d'emblèmes, antérieurs ou postérieurs, militaires ou civils.

Parmi les différents systèmes utilisés par les sociétés non occidentales, ce sont probablement les mon japonais qui présentent avec les armoiries européennes les ressemblances les plus étroites. Comme en Occident, c'est dans le courant $\mathrm{du} \mathrm{XII}^{\mathrm{e}}$ siècle que ces emblèmes, d'abord individuels et 
d'origine militaire (se faire reconnaître au combat), opèrent leur greffe sur la parenté, et deviennent familiaux et héréditaires: tous les membres d'un même clan portent désormais le même mon. Comme les armoiries occidentales, ces mon disent à la fois l'identité et la parenté, servent de marques de possession et remplissent des fonctions ornementales. Par là même, ils prennent place sur de nombreux supports, notamment les vêtements, les étoffes, les armes et les objets. Toutefois, entre les armoiries européennes et les mon japonais, il existe aussi de fortes différences, qu'il ne faut pas dissimuler. Les couleurs, par exemple, qui jouent un rôle essentiel dans les armoiries, ne comptent pas, ou guère, pour les mon; ceux-ci prennent la couleur du support sur lequel ils sont apposés. De même, le répertoire des figures est fort différent: végétal à $80 \%$ au Japon, beaucoup plus diversifié en Europe (animaux, végétaux, objets, figures géométriques). Enfin, alors que le mon est commun à l'ensemble d'un clan et concerne un grand nombre de personnes, les armoiries disent souvent l'individu et précisent sa place au sein du groupe familial.

Je n'ai pas les compétences nécessaires pour faire ici une véritable étude comparée des armoiries et des mon. Ce devrait du reste être la tâche d'un travail collectif. Mais profitant du thème du présent volume, je souhaiterais attirer l'attention sur une catégorie d'armoiries particulière, très en vogue au Moyen Âge dans toute l'Europe: les armoiries «parlantes». Il s'agit d'une véritable écriture en images.

\section{Une définition difficile}

Définir les armoiries parlantes n'est pas un exercice aisé, car elles s'expriment par des formules variées. Grossièrement on peut dire que sont «parlantes» les armoiries dans lesquelles le nom de certains éléments - le plus souvent celui de la figure principale - forme un jeu de mots ou établit une relation de sonorité avec le nom du possesseur de l'armoirie. Le cas le plus simple est celui où le nom de la figure principale et le nom du possesseur entretiennent une relation directe, comme par exemple sur trois sceaux normands du XIII ${ }^{\text {e }}$ siècle: Jean de La Tour porte une tour; Baudouin de Corbeil, un corbeau; Raoul Cuvier, un cuvier (fig. 1) ${ }^{1}$.

Mais il existe des liens entre le nom et la figure qui sont moins patents. La relation peut être allusive (ainsi, toutes les familles dont le nom évoque une porte et qui placent des clefs dans leurs armes) ou bien se faire avec une partie du nom seulement: Guillaume de Capraville, puissant baron anglais, place dans son écu, vers 1300 , une simple chèvre ${ }^{2}$, tandis que, quelques décennies plus tard, Pierre d'Orgemont, chancelier de France (1373-1380) arbore fièrement trois épis d'orge ${ }^{3}$. La relation peut également être construite 
Une écriture en images: les armoiries parlantes

Figure 1. Une figure sigillaire parlante: un cuvier. Sceau de Raoul Cuvier, paysan normand (début du XIII ${ }^{\mathrm{e}}$ siècle). Paris, Arch. Nat., sceau D 4203.

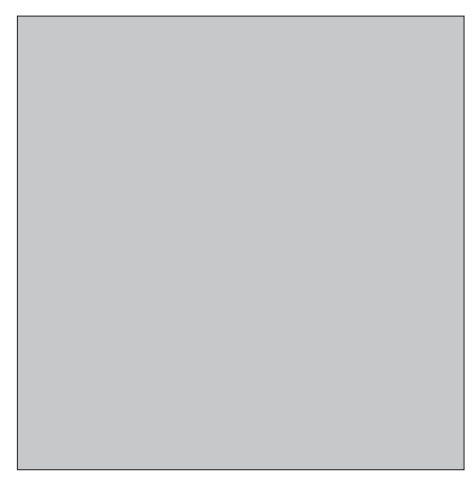

sur le nom d'une couleur et non pas d'une figure: la grande famille florentine des Rossi porte ainsi un écu tout rouge (de gueules plain $)^{4}$. Ou bien, et plus fréquemment, elle peut être construite sur les noms associés de plusieurs figures et former une sorte de rébus: les comtes de Helfenstein, par exemple, largement possessionnés dans le nord de la Suisse et dans le Wurtemberg, associent dans leurs armes un éléphant (Elefant) et un rocher (Stein) (pl. 29, p. 111); les Chiaramonte, originaires de Vérone, un mont surmonté d'une étoile qui semble l'éclairer. Exceptionnellement, la relation parlante se fait non pas avec le nom de famille mais avec le nom de baptême. Citons pour exemple l'écu gironné d'or et d'azur d'un shériff du Northampshire nommé Giron de Bassingburne, écu blasonné par plusieurs armoriaux de la seconde moitié du XIII ${ }^{\mathrm{e}}$ siècle ${ }^{5}$.

La notion de «jeu de mots» est elle-même très floue, ou du moins évolue avec le temps: ce qui constitue un jeu de mots au XIII ${ }^{\mathrm{e}}$ siècle peut ne plus être perçu ou considéré comme tel au XV $\mathrm{VV}^{\mathrm{e}}$ ou au XVII ${ }^{\mathrm{e}}$ siècle. D'où la difficulté qu'il y a à définir de manière univoque ces armoiries que le français et l'allemand qualifient de «parlantes» (redende Wappen). L'anglais, plus poétique ou plus précis, les nomme joliment «chantantes» (canting arms). Cette expression, qui insiste sur l'harmonie sonore de la relation entre le nom de la personne et celui de la figure, se retrouve dans la langue latine des érudits du XVII ${ }^{\mathrm{e}}$ siècle: arma cantabunda ou arma personantia ${ }^{6}$.

\section{Une pratique ancienne}

Malgré leur grand intérêt, les armoiries parlantes attendent encore leurs historiens. Ni les héraldistes (trop peu philologues), ni les philologues (trop peu héraldistes), ni les anthroponymistes ne s'y sont jusqu'à présent véritablement intéressés ${ }^{7}$. Le matériel est pourtant abondant et suscite de 
nombreuses interrogations. Mais il semble que ces armoiries souffrent encore du discrédit dans lequel les ont tenues les héraldistes du XVII et du XIX ${ }^{\mathrm{e}}$ siècle. Elles passent pour moins anciennes, moins nobles et, héraldiquement, moins pures que les autres armoiries ${ }^{8}$. Ce qui est absolument faux. Les armoiries parlantes existent depuis la naissance de l'héraldique au XII ${ }^{\mathrm{e}}$ siècle, et à cette date de très grandes familles en ont fait déjà usage: les comtes de Bar (deux bars adossés), les comtes de Boulogne (trois «boules» ou tourteaux) (fig. 2, p. 192), les comtes de Minzenberg (une branche de menthe), les sires de Hammerstein (un marteau) et bien d'autres, sans compter le royaume de Castille (des châteaux) et celui de Léon (un lion). Au reste, les hérauts d'armes du Moyen Âge, quand ils ne connaissent pas les armoiries d'un royaume (réel ou imaginaire) ou d'une grande seigneurie, n'hésitent pas à forger des armoiries parlantes pour pallier les lacunes de leur information. De telles armes leur semblent naturelles et parfaitement fidèles à l'esprit du blason. C'est ainsi qu'un héraut d'armes français, compilant un armorial universel aux environs des années 1300, attribue au roi de Portugal un écu ayant pour figure une porte (pl. 30, p. 112), au «roi» de Galice un écu orné d'un calice et au prétendu roi du Maroc un écu à trois rocs d'échiquier (ibid). Ces armes imaginaires connaissent un certain succès et sont reprises par d'autres armoriaux du XIV siècle, y compris des armoriaux germaniques pour lesquels la relation parlante entre les noms et les figures n'est pourtant plus intelligible ${ }^{9}$.

D'une manière générale, les armoiries parlantes ne sont ni moins anciennes, ni moins honorables, ni moins héraldiques que les autres. Mais leur abondance dans l'héraldique roturière à partir de la fin du Moyen Âge et les jongleries verbales sur lesquelles elles sont quelquefois construites à l'époque moderne les ont déconsidérées aux yeux des héraldistes de l'Ancien Régime. Ceux d'aujourd'hui ne peuvent plus les tenir dans le même discrédit; ils doivent les étudier comme d'authentiques documents d'histoire culturelle et s'efforcer d'en cerner la diffusion, d'en comprendre les mécanismes et les enjeux.

\section{Un repérage difficile}

Cependant, apprécier pour chaque époque, chaque région, chaque classe ou catégorie sociale la proportion des armoiries parlantes par rapport à l'ensemble des armoiries n'est pas un exercice facile. Surtout pour la période médiévale. Cette proportion est toujours sous-évaluée parce que souvent nous ne savons pas reconnaître de telles armes. Tantôt la relation entre le nom et la figure est plus allusive que proprement «parlante», et ce qui était limpide ou ingénieux pour nos ancêtres ne l'est plus 
Une écriture en images: les armoiries parlantes

nécessairement pour nous. Tantôt elle se construit sur des termes dialectaux ou bien sur des mots disparus. Plusieurs familles Fouquet, dont celle du surintendant général des finances de Louis XIV, portent ainsi un écureuil dans leurs armes parce que fouquet est un des noms désignant cet animal en moyen français. De même, de nombreuses armoiries bretonnes construisent la relation parlante non pas sur le français ou sur le latin, mais sur le breton: les seigneurs d'Avaugour, par exemple, placent dès les années 1260 un pommier dans leurs armes parce qu'en breton aval gor signifie «pomme sauvage». En Angleterre, c'est souvent l'anglo-normand qui explique le choix de telle ou telle figure, choix inintelligible si l'on part de l'anglais. Citons quelques exemples. Dès la fin du XII ${ }^{\mathrm{e}}$ siècle, la grande famille anglaise des Lucy porte dans ses armes trois brochets: la relation parlante n'est perceptible que si l'on sait que «brochet» (qui en anglais moderne se dit pike) se dit en anglo-normand lus. De même, dès le XIII ${ }^{\mathrm{e}}$ siècle, on peut voir dans les armes des Maulevrier, possessionnés dans le nord de l'Angleterre, un lévrier (greyhound); dans celles des Luttrel, une loutre (otter); dans celles des de Vele, un veau (calf); et dans celles des Fitz-Urse, un ours (bear). Pour comprendre de tels choix, il faut remonter aux origines normandes de ces puissantes familles et connaître le lexique anglo-normand en usage dans l'Angleterre féodale.

Dans les exemples qui viennent d'être cités, la figure parlante est un animal. Retrouver la relation existant entre le nom de cet animal et le nom du possesseur de l'armoirie est parfois aisé, parfois plus difficile, jamais vraiment impossible. Mais quand il s'agit d'une figure géométrique, la relation parlante est souvent moins directe ou moins limpide, et au problème de la langue s'ajoute celui du degré de relation ou d'allusion. Lorsqu'en 1265 Guillaume des Barres, simple chevalier, orne le champ de son sceau d'un écu losangé, c'est-à-dire d'un écu semé de losanges formant comme un grand filet ${ }^{10}$, la relation parlante est malaisée à deviner; elle existe pourtant: le losangé évoque des «barres», c'est à dire un obstacle. Un peu plus tard, les Montagu, comtes de Salisbury, présentent dans leurs armes une figure parlante formée d'une fasce de fusées, c'est-à-dire une figure géométrique faite de losanges très pointus dont chaque sommet ressemble à un «mont aigu».

De telles combinaisons entre le nom et la figure existent dès l'apparition des premières armoiries. En fait, il semble bien qu'au XII siècle, la jeune héraldique ait hérité des sceaux et des monnaies cet usage des emblèmes parlants. Avant même d'adopter des armoiries, quelques-uns des grands lignages cités plus haut faisaient déjà représenter des figures parlantes dans le champ de leurs sceaux ou dans celui des monnaies qu'ils faisaient frapper. Au tournant des $\mathrm{XI}^{\mathrm{e}}-\mathrm{XII}^{\mathrm{e}}$ siècles, c'est déjà le cas des comtes de Bar, des comtes de 
Minzenberg, des comtes de Boulogne. Les trois «boules» de ces derniers se voient même sur la broderie de Bayeux, à l'horizon des années 1080, soit plus d'un demi-siècle avant l'apparition des premières armoiries: dans une scène figurant la bataille d'Hastings, Eustache II comte de Boulogne, allié du duc Guillaume, porte un gonfanon déjà orné des trois bollae (bullae), emblèmes parlants du comitatus Boloniensis (fig. 2) ${ }^{11}$.

Ce témoignage est évidemment capital pour étudier la genèse des armoiries et la protohistoire de l'héraldique. Toutefois, on pourrait remonter plus haut encore et observer que l'usage d'emblèmes familiaux parlants a déjà cours dans l'Antiquité romaine: sous la République, par exemple, la gens Porceia a pour emblème un sanglier et la gens Calpurnia, l'image d'un cheval au galop (en grec kalpis) ${ }^{12}$. Ou bien, sans remonter aussi loin, souligner que, dès le haut Moyen Âge, des saints ont reçu pour attribut iconographique une figure formant un jeu de mots avec leur nom: un agneau pour sainte Agnès dès l'époque paléochrétienne; plus tard un coq (gallus) pour saint Gall, un loup pour saint Loup, un ours pour saint Ursin et sainte Ursule; plus tard encore un lion pour saint Léonard, un chat (felis) pour saint Félix, une peau d'Africain (maurus) pour saint Maurice.

Figure 2. Gonfanon pré-héraldique aux trois «boules» du comte Eustache II de Boulogne à la bataille d'Hastings. Broderie de Bayeux, vers 1070 . 
Une écriture en images: les armoiries parlantes

\section{Une spécialité germanique?}

Sur l'ensemble des armoiries médiévales publiées à ce jour, au moins $20 \%$ peuvent être reconnues comme parlantes, à un titre ou à un autre. Mais cette proportion est certainement inférieure à la réalité puisque la relation parlante entre le nom et tel ou tel des éléments composant l'armoirie ne se laisse pas toujours reconnaître. Chronologiquement, il semble que cette proportion soit plus grande encore à la fin du Moyen Âge, lorsque beaucoup de roturiers et de communautés se dotent d'armoiries. C'est le procédé le plus simple pour se choisir une figure héraldique. Les villes, par exemple, y ont fréquemment recours: une fleur de lis pour Lille (dès la fin du XII siècle) et pour Florence, un ours (Bär) pour Berne et pour Berlin, un lion pour Lyon, une roue de moulin pour Mulhouse, trois rats pour Arras, etc. Les exemples en sont nombreux. Quant aux corps de métiers, la figure est en général plus allusive que parlante: un bœuf ou un porc pour les bouchers, un champ de vair pour les pelletiers, des ciseaux pour les tailleurs, mais faire ici une distinction nette entre emblème parlant et emblème allusif n'est pas toujours aisé ni pertinent.

Géographiquement les armoiries parlantes se rencontrent partout, mais il semble bien que dans les pays germaniques elles soient plus nombreuses que dans tout autre région. À cela des raisons à la fois linguistiques et culturelles. La langue allemande et, d'une manière générale, les langues germaniques semblent mieux se prêter à jouer ainsi sur les mots. En outre, l'anthroponymie germanique sollicite plus directement que l'anthroponymie romane les noms d'animaux, de végétaux, de couleurs ou d'objets; ou du moins la relation entre le nom et la chose y semble plus claire, plus facile à exprimer et à reconnaître. Enfin, l'usage de figures parlantes paraît avoir eu en Allemagne et dans les pays germaniques meilleure presse que dans le reste de l'Europe. C'est peut-être pourquoi on en a usé et abusé. Aux XIV et XV siècles, par exemple, alors que quelques grandes familles françaises, espagnoles ou italiennes cherchent à dissimuler l'origine parlante de leurs figures héraldiques et inventent des légendes héroïques pour en expliquer la genèse et la signification - le cas des Visconti est le plus fameux ${ }^{13}$ - les familles comtales allemandes ou autrichiennes n'ont aucunement honte de leurs emblèmes parlants et sont fières de souligner le lien qui unit le nom et la figure. Ce lien n'est aucunement perçu comme dévalorisant: les comtes de Henneberg sont ainsi fiers de montrer leur poule (Henne) perchée sur un mont (Berg) (pl. 31, p. 112); les comtes de Tierstein s'amusent à changer l'animal (Tier) présent dans leurs armes : tantôt c'est une biche, tantôt un chien, parfois un loup ou un mouton, mais cet animal instable est toujours posé sur une pierre (Stein) afin que les deux figures associées forment un rébus parlant; 
quant aux célèbres Wolkenstein, puissants seigneurs du Tyrol qui ont donné deux poètes, ils mettent partout en scène leur curieux écu tranché-nébulé, c'est-à-dire divisé obliquement par une ligne en forme de nuages (Wolken). Mieux que toute autre formule, l'armoirie parlante - véritable procédé mnémotechnique - exprime la mémoire et la cohésion du lignage, articulées autour d'un nom pleinement assumé et exprimées par une ou plusieurs figures qui constituent un authentique patrimoine emblématique.

C'est du reste dans les pays de langue allemande qu'à la même époque bon nombre de saints reçoivent pour attributs ces figures, déjà évoquées, formant des jeux de mots plus ou moins approximatifs avec leur nom. Parfois la relation parlante ne s'établit pas seulement entre le nom du saint et son attribut, mais entre son nom et le mal qu'il est censé guérir: saint Gall, par exemple, est invoqué pour guérir les maladies à bubons (die Gallen), saint Blaise pour réduire les ampoules (die Blasen) et saint Augustin, pour soulager les troubles des yeux (die Augen) - maux pour lesquels en France on sollicite respectivement saint Maclou (clous), saint Ampère (ampoules) et sainte Claire (idée de clarté).

De telles pratiques et croyances n'ont rien d'anecdotique ni de circonstanciel. Elles se situent, au contraire, au cœur de la symbolique médiévale et mettent en valeur l'immense pouvoir des noms, propres ou communs, dans la construction des savoirs et dans les systèmes de valeurs qui s'y rattachent.

\section{De la figure au nom et retour}

Pour terminer cette évocation des armoiries parlantes à l'époque médiévale, il reste deux problèmes importants à évoquer. Le premier est lié à la genèse des noms de famille entre le $\mathrm{XI}^{\mathrm{e}}$ et le XIII ${ }^{\mathrm{e}}$ siècle. Dans plusieurs cas, il apparaît bien que ce n'est pas l'emblème - héraldique ou pré-héraldique qui s'est construit sur le nom, mais qu'au contraire c'est le nom qui est né de l'usage répété d'un emblème, le plus souvent collectif, jouant un rôle plus ou moins «totémique» (j'emploie évidemment ce mot dans un sens galvaudé par rapport à l'usage qu'en font les anthropologues). Autrement dit, dans certains pays et dans certaines sociétés, l'emblème familial précède le nom et lui donne naissance. Les exemples en sont nombreux en Hongrie et en Pologne ${ }^{14}$. Mais ils existent aussi en Italie, en Écosse et en Suède - la grande famille des Oxenstierna, par exemple, a longtemps scellé d'une tête de boeuf (Ochs) avant de recevoir son nom au $\mathrm{XIV}^{\mathrm{e}}$ siècle ${ }^{15}-$, trois pays où l'héraldique est longtemps restée greffée sur la parenté large et n'était pas construite sur la parenté étroite, comme en France ou en Angleterre. Mais dans ces deux 
derniers royaumes, ainsi qu'en Allemagne et en Espagne, il est probable que des cas semblables, sans doute moins faciles à discerner, existent aussi. Pensons simplement au surnom prestigieux des «Plantegenêt», né de l'usage (réel ou fictif, peu importe) d'un emblème en forme de branche de genêt par leur ancêtre éponyme Geoffroi le Bel, comte d'Anjou et duc de Normandie, mort en $1151^{16}$. Pensons aussi aux emblèmes héraldiques qui aident à distinguer deux branches d'une même famille et qui donnent à chacune son nom complet: ainsi chez les riches banquiers Fugger d'Augsbourg; dès le milieu du XV $\mathrm{XV}^{\mathrm{e}}$ siècle, «les Fugger au daim» sont distingués des «Fugger au lis» par une formule anthroponymique construite sur la figure héraldique de chacune des deux branches. Pensons enfin à des exemples plus récents comme le nom des Rothschild, tardivement formé à partir de l'enseigne de leur maison de Francfort (zum roten Schild: à l'écu rouge). Des enquêtes approfondies sur ces questions essentielles seraient les bienvenues.

L'autre problème touche aux systèmes de valeurs médiévaux. On constate en effet que certaines familles ne portent pas d'armoiries parlantes alors que leur nom s'y prêterait facilement. Parfois, cette absence d'armoiries parlantes est liée à l'histoire héraldique primitive de la famille: des armes de fief, liées à la terre et non pas au nom, ont pu perdurer et rendre inutile la création d'une armoirie parlante; ou bien la famille a simplement repris les armes de son seigneur en en changeant les couleurs (cas fréquent dans l'est de la France et en Flandre). Mais dans d'autres cas, plus nombreux, c'est parce que la figure qui aurait pu constituer un emblème parlant était par trop péjorative qu'elle a été rejetée. Ainsi les familles (ou les individus) dont le nom évoque le chat - comme la puissante famille souabe des Katzenellenbogen, ou bien celles des seigneurs néerlandais van Katse - ont préféré prendre pour emblème un autre animal (loup, léopard) ou une autre figure parce que le chat, jusqu'au $\mathrm{XIV}^{\mathrm{e}}$ siècle, est perçu comme une créature diabolique. Même renoncement fréquent devant le porc (domestique), le renard, le crapaud, le serpent et d'autres animaux prenant place dans le bestiaire du Diable. Il est alors permis de se demander pourquoi l'anthroponymie fait appel à de telles figures tandis que l'héraldique les évite. Contrairement à l'armoirie, le nom de famille estil dès l'origine un emblème totalement subi et non pas choisi?

\section{Le danger de l'anachronisme}

Les armoiries parlantes ne disparaissent pas avec le Moyen Âge, bien au contraire. À partir de la fin du XVI ${ }^{\mathrm{e}}$ siècle, elles se multiplient et prennent un aspect plus mécanique et plus systématique. Les créateurs ne cherchent plus dans les mots la vérité des êtres et des choses, mais s'amusent simplement à 
jouer sur les mots pour susciter l'attention, le rire ou la dérision. Au $\mathrm{XVII}^{\mathrm{e}}$ siècle, le grand-père de Jean Racine, l'illustre poète, reçut ainsi un écu composé d'un rat et d'un cygne! Dans les armoiries roturières, qui sont alors de plus en plus nombreuses et de plus en plus souvent représentées sur des objets, des images et des bâtiments de toute nature, les figures parlantes deviennent envahissantes, au point de faire croire en certaines régions (l'Allemagne méridionale, la Suisse, l'Italie du Nord) que l'héraldique est presque entièrement construite autour de ces dernières.

Soulignons pour conclure combien l'histoire de ces armoiries parlantes, envisagée dans la longue durée, soulève des problèmes complexes et trop peu étudiés. Ils sont tout ensemble liés à la formation et à la perception des noms propres, à la symbolique des sons, à l'histoire des corrélations analogiques et aux constantes préoccupations étymologiques des sociétés européennes. Par là même, ils mériteraient une réflexion collective, réunissant des héraldistes, des philologues et des historiens, ainsi que des travaux renouvelés à la lumière des acquis récents des études anthroponymiques et des recherches en cours sur les parétymologies. Ils mériteraient également d'être comparés avec des pratiques semblables existant dans l'Antiquité ou dans des sociétés non occidentales (existe-t-il au Japon des mon «parlants»?). Mais leur étude nécessite aussi beaucoup de prudence, car sur ces terrains difficiles le danger de l'anachronisme guette l'historien à chaque coin de document. Il lui est en effet impossible de projeter tels quels dans le passé les savoirs, les définitions, les classements et les modes de sensibilité qui sont les nôtres aujourd'hui. Ce n'étaient pas ceux d'hier ni d'avant-hier. Les systèmes de valeurs et les formes d'expression autour desquels se construisent et fonctionnent les armoiries parlantes varient dans l'espace et dans le temps : l'humour, l'ironie, le jeu de mots, le calembour, l'assonance, l'allitération et même les notions d'analogie et d'étymologie ne sont pas les mêmes au XII ${ }^{\mathrm{e}}$ siècle, au XV ${ }^{\mathrm{e}}$, au $\mathrm{XVII}^{\mathrm{e}}$ et de nos jours. L'historien doit constamment s'en souvenir.

\section{NOTES}

1. Louis Douët d'Arcq, Archives de l'Empire. Inventaires et documents. Collection de sceaux, Paris, 1863-1868, 3 vol., vol. 1, n 3733, 1887, 4203.

2. Gerald J. Brault, Early Blazon. Heraldic Terminology in the Twelfth and Thirteenth Centuries With Special Reference to Arthurian Literature, Oxford, Oxford University Press, 1972, p. 36.

3. Douët d'Arcq, op. cit., vol. 1, n 3113.

4. Michel Popoff, Répertoires d'héraldique italienne I: Florence (1302-1700), Paris, Références, 1991, p. 11 (A 388).

5. Cecil R. Humphery-Smith, Anglo-Norman Heraldry and Related Studies, 
Canterbury, 1973, p. 69, n 103.

6. Ces expressions latines ne semblent pas antérieures au XVII ${ }^{\mathrm{e}}$ siècle, tandis que l'expression française «armes parlantes» se rencontre dès la fin du XIV siècle. Notons que les auteurs anglais utilisent parfois l'expression punning arms au lieu de canting arms.

7. Il n'existe aucune étude scientifique sur la question des armoiries parlantes. Outre les paragraphes consacrés à cette question dans les principaux manuels et traités d'héraldique, on lira: Max Prinet, «Cimiers et supports parlants d'armoiries françaises », dans Archives héraldiques suisses, 1916, p. 12-21; Winifried Hall, Canting and Allusive Arms of England and Wales, Canterbury, Achievements, 1966; Jorge Preto, Des armoiries qui parlent: propos sur la science du blason et la linguistique, Lisbonne, Instituto Português de Heráldica, 1986 (cette dernière brochure est quelque peu ésotérisante et contient de nombreuses erreurs; mais elle cite plusieurs exemples espagnols et portugais originaux).

8. C'est l'opinion de tous les auteurs français et anglais du XIX ${ }^{\mathrm{e}}$ siècle. Un témoignage prolongé de ce discrédit des armoiries parlantes à l'époque contemporaine est fourni par l'héraldique urbaine: beaucoup de petites villes françaises dont le nom s'associerait facilement avec une figure parlante refusent d'adopter une telle figure lorsqu'elles se font créer des armes. Elles ont l'impression que cette relation parlante est plus ou moins ridicule et fort peu héraldique. Idées fausses mais idées indélébiles, malheureusement. Cette réticence devant les emblèmes parlants se retrouve semblablement dans le monde des logos, du moins en France.

9. Voir Walther Merz \& Friedrich Hegi, Die Wappenrolle von Zürich. Ein heraldisches Denkmal des vierzehnten Jahrhunderts in getreuer farbiger Nachbildung des Originals mit den Wappen aus dem Hause zum Loch, Zurich, Orell Füssli, 1930, n 10 et 11.

10. Douet d'Arcq, op. cit., vol. 1, n 1298.

11. Michel Pastoureau, Traité d'héraldique, Paris, Picard, 1979, p. 298-299.

12. Voir d'autres exemples cités par Ernest Babelon, Description historique et chronologique des monnaies de la République romaine vulgairement appelées monnaies consulaires, 2 vol., Paris, Rollin et Feuardent, 1885, vol. 1, p. XLVI-L, et par Michael H. Crawford, Roman Republican Coinage, London/New York, Cambridge University Press, 1974, p. 725-734.

13. Les Visconti, ducs de Milan et comtes de Pavie, ne sont à l'origine que seigneurs d'Anguera, terre dont le nom évoque celui du serpent (anguis). Il est probable que leur célèbre figure héraldique en forme de «guivre» (serpent vomissant un enfant) est au départ une figure parlante avec le nom de cette terre. Mais dès le milieu du XIV ${ }^{\mathrm{e}}$ siècle est forgée une légende hérö̈que expliquant que cette guivre a été tuée par le fondateur du lignage, revenu de croisade au moment même où son fils venait d'être enlevé et englouti par ce serpent monstrueux. Il parvint à le vaincre et à lui faire vomir l'enfant. Voir Académie internationale d'héraldique (éd.), Le cimier : mythologie, rituel, parenté, des origines au XVI ${ }^{e}$ siècle, Actes du $6^{\mathrm{e}}$ colloque international d'héraldique, Bruxelles, 1990, p. 360, note 22.

14. Szabolcs de Vajay, L'Héraldique hongroise, Lausanne, 1961, p. 20; Stefan 


\section{Michel Pastoureau}

K. Kuczynski, Polskie herby ziemskie: geneza, treści, funkcje, Varsovie, 1993, p. 39-67.

15. John Woodward et George Burnett, A Treatise on Heraldry British and Foreign, Edinburgh/London, W. \& A.K. Johnston, 1896 (2 éd.), p. 671-672.

16. Sur l'origine du nom Plantegenêt et sur l'emblème de Geoffroi, voir, faute de mieux, C. Wilfrid Scott-Giles, The Romance of Heraldry, Londres, J. M. Dent, 1965 (nouv. éd.), p. 49-50. 